\title{
On Modeling Clustering Indexes of BT-like Systems
}

\author{
Q.H. Li John C.S. Lui \\ The Chinese University of Hong Kong
}

\begin{abstract}
In this paper, we explore the "clustering" phenomenon in BT-like systems. A high clustering implies peers have a high tendency to exchange information with peers of the similar bandwidth type. We first show the clustering does exist in BT-like systems. Although high clustering is desirable for file sharing application, it may not be appropriate for multimedia streaming applications. We provide analytical models to calculate the clustering index and illustrate how one can control the clustering index for different P2P applications.
\end{abstract}

\section{Introduction}

In recent years, there is a significant increase of $\mathrm{P} 2 \mathrm{P}$ traffic, in particular, due to the BitTorrent (BT) protocol. In fact, we have seen a growing applications, for example, file sharing, content distribution, multimedia streaming and videoon-demand,..etc, all use the BT-derivative protocols to provide high performance and scalable service.

The BT protocol adopts the well known tit-for-tat policy within the choking algorithm for peer selection. Peers in such a system will reward other peers who contribute more and minimize the upload service to the free-riding peers. Thus, every peer has incentives to upload in order to enjoy a better service. There are a number of papers [1], [6] which provide the quantitative analysis on BT's performance and fairness. However, most of the work do not capture the detail of the choking algorithm, which influence how peers select other peers for information exchange. Since the tit-for-tat policy affects the preference of peer selection, it can induce the clustering phenomenon within a BT-like system, e.g., peers which are of similar bandwidth type may prefer to exchange information with each other. When a BT-like file distribution system has a high clustering index, it implies that peers of type $k$ may prefer to exchange information with peers of type $k$, and intuitively, this provides certain degree of fairness (since similar bandwidth peers exchange information). However, for some other BT-like systems, e.g., multimedia streaming or video-on-demand service, one may not want the system to have high clustering since we want a more resourceful peers to stay in the system to help other less resourceful peers to receive the information on time.

The goal of this paper is to investigate the clustering phenomenon of BT-like system. We provide an analytical model to evaluate the clustering index of a BT-like system which uses the greedy selection algorithm (a derivative of the titfor-tat policy). In particular, we examine in detail the choking algorithm and analyze the peer's behavior in a heterogeneous system. Unlike the work in [3], which investigate the clustering via measurement, we provide a general analytical model to understand this phenomenon and we also show how to use our model to control the degree of clustering so as to adapt to the requirements of different $\mathrm{P} 2 \mathrm{P}$ applications. The contributions of this paper are:

- We develop two analytical models, one for BT-like systems with two types (or groups) of peers, while the other for BT-like system with many types, in estimating the clustering index among peers.

- We validate our models extensively via simulation.

- We propose simple knobs to control the clustering index of a BT-like system.

In Section II, we introduce the peer selection algorithm, in Section III, we present our analytical models. Performance evaluation and validation are presented in Section IV and Section V concludes.

\section{Peer Selection Algorithm}

In this paper, we consider a particular peer selection algorithm, or the Greedy Selection (GS) algorithm, which is based on the classification of peer's uploading bandwidth. This algorithm will help us to understand how peer selection may impact the clustering phenomenon in BT-like systems. We assume that there are $n>1$ different groups of peers, with group $k$ denoted as $G_{k}$ and $G=\bigcup_{k=1}^{n} G_{k}$, with $G_{k} \bigcap G_{l}=\emptyset$ for $k \neq l$. Peers in $G_{k}$ has an upload capacity of $U_{k}$. Without loss of generality, we assume $U_{1}>U_{2}>\cdots>U_{n}$. Each peer in the system can provide $K$ maximum uploads to other peers. i.e, in the BT protocol, $K$ is set to five. We assume throughout this paper that the capacity of an uploading connection is limited to $u_{k}=U_{k} / K$ for any peer in group $G_{k}$. Our system follows the uplink sharing model [5] wherein the bandwidth constraint is in the uplink connections rather than the downlink connections. Note that this assumption is true for most broadband access technologies.

The Greedy Selection algorithm, which is also known as the tit-for-tat in the official BitTorrent protocol, is an effective policy to discourage free riding. A peer using this algorithm will unchoke (which means to provide upload service) the top $K-1$ peers who contribute the most downloading service to this peer. These $K-1$ connections are also called the regular unchoking connections and this operation is carried out every $10 \mathrm{sec}$. The remaining upload connection will randomly select another peer in the system to unchoke. This occurs every 30 sec and it is called the optimistic unchoking. The purpose of the optimistic unchoking operation is to discover peers which may be able to provide better downloading service to this peer. The regular unchoke is greedy oriented since it only reciprocates peers who contribute more service to this peer, while the optimistic unchoke serves as a discovery mechanism 
to find a more contributive peer [1], [3]. We model the GS algorithm as follows. Each round there is a regular unchoke event, while every $\omega \geq 1$ rounds there is an optimistic unchoke event. At the end of each round, the following operations will be carried out:

1) A peer sorts all its contributing peers in the current round according to their group number (e.g., for bandwidth differentiation). If there are several contributing peers belonging to the same group, then the contributing peer which provides upload service for a longer period will have a higher priority.

2) The peer will unchoke at most $K-1$ contributing peers which have the highest $K-1$ priority.

3 ) Every $\omega$ rounds, this peer randomly picks one neighboring peer, independent of its group number or service contribution, to provide optimistic unchoking service.

For example, consider the system with two groups of peer, $G_{1}$ and $G_{2}$. A peer belongs to $G_{1}$ and it has $K=5$ upload connections. If there are two contributing peers from $G_{1}$ and one from $G_{2}$, then this peer will provide three regular unchoke connections: two to the contributing peers in $G_{1}$ and one to the contributing peer in $G_{2}$. This peer also uses one upload connection for optimistic unchoke, and one of its upload connections will remain idle for the current round.

It is important for us to point out that for the GS algorithm, when a peer provides a regular unchoke to another contributing peer, then a bi-directional connection is established since both peers provide upload service to each other. On the other hand, for the optimistic unchoke service, we only have a unidirectional connection.

\section{Analytical Models}

In this section, we first provide the definition of clustering index, then we will show how to formulate the mathematical model to estimate the clustering index of BT-like systems. To simplify our presentation, we first show how one can use a Markov chain to model the Greedy Selection algorithm with two groups of peers in a close system, that is, all the peers arrive at the same time and they will not leave. In this BTlike system, we have $N$ peers with $N_{1}$ and $N_{2}$ being the number of peers in group $G_{1}$ and $G_{2}$ respectively. When extending the model for more than two groups, the state space and correspondingly, the one-step transition probability matrix becomes large. To resolve the curse of state space explosion, we simplify the state space and propose another Markovian model to represent the Greedy Selection algorithm so that we can derive the clustering effect for BT-like systems with more than two peer groups. Throughout this paper, we denote $B(N, p, k)$ as the binomial probability $\left(\begin{array}{c}N \\ k\end{array}\right) p^{k}(1-p)^{(N-k)}$. Unless we state otherwise, we set $\omega=1$ (i.e., at each round, each peer will have an optimistic unchoke operation).

\section{A. Clustering Index}

Clustering index is a measure of the fraction (or steady state probability) of bi-directional connections between peers of the same group. For example, given that $K$ is the number of upload connections of a peer and $K-1$ of these upload connections are used for regular unchokes, and if all of these $K-1$ upload connections are used to connect to other peers of the same group, then the clustering index will be 1 . There are two important points to note: (1) regular unchokes will create a bi-direction connection between two peers from the same group; (2) a higher clustering index implies a higher tendency for peers of similar bandwidth type to exchange information.

Assume that a peer $m$ of group $G_{k}$, we define $c_{m}$ to be the clustering index for peer $m$ as:

$$
c_{m}=\frac{\# \text { of bi-directional connections to peers in } G_{k}}{K-1} .
$$

The clustering index for peers in $G_{k}$, where $1 \leq k \leq n$ is:

$$
C_{k}=\frac{\sum_{m \in G_{k}} c_{m}}{\left|G_{k}\right|}
$$

where $\left|G_{k}\right|$ is the number of peers in group $G_{k}$.

It is easy to verify that $C_{k} \in[0,1]$. When $C_{k}$ is larger than the fraction of $G_{k}$ peers in the system, then a tighter cluster forms. This means that the unchokes from peers of $G_{k}$ will have a higher priority and peers in $G_{k}$ tend to unchoke their compeers (e.g. other peers in $G_{k}$ ). On the other hand, if $C_{k}$ is smaller than the fraction of $G_{k}$ 's peers in the system, then the unchokes from $G_{k}$ is less competitive and peers tend to unchoke other groups which may provide a better service.

\section{B. Analysis of the Greedy Selection Algorithm}

Given the description of the GS algorithm from Section II, we can construct a discrete time Markov chain $\mathcal{M}_{G}$ with the following state space $S_{G}$ :

$$
S_{G}=\{(i, j) \mid i \geq 0, j \geq 0, i+j \leq K-1\},
$$

where $i$ is the number of bi-directional connections (or regular unchokes) to group $G_{1}$ and $j$ is the number of bi-directional connection to group $G_{2}$. We also define the following probability vectors:

$$
\boldsymbol{\pi}^{(1)}=\left\{\pi_{i, j}^{(1)} \mid(i, j) \in S_{G}\right\}, \quad \boldsymbol{\pi}^{(2)}=\left\{\pi_{i, j}^{(2)} \mid(i, j) \in S_{G}\right\},
$$

where $\pi_{i, j}^{(k)}$ represents the fraction of group $G_{k}$ peers in state $(i, j)$, for $k=\{1,2\}$. Follow the definition of clustering above, the following expressions are the clustering index for $G_{1}$ and $G_{2}$ :

$$
C_{1}=\frac{\sum_{(i, j) \in S_{G}} i \pi_{i, j}^{(1)}}{K-1}, \quad C_{2}=\frac{\sum_{(i, j) \in S_{G}} j \pi_{i, j}^{(2)}}{K-1} .
$$

In the GS algorithm, a bi-directional connection between two peers in $G_{1}$ will hold once it is established since they have the highest selection priority under the GS algorithm. A bi-directional connection between a peer in $G_{1}$ and a peer in $G_{2}$ can be terminated when the peer in $G_{1}$ receives a new optimistic unchoke request from a compeer (e.g., another peer in $G_{1}$ ). While the bi-directional connection between two peers in $G_{2}$ can be terminated if a peer from $G_{1}$ has an optimistic unchoke request to either of these two peers. Lastly, there are other factors that will terminate a bi-directional connection, 
e.g., when the file is not available or when network errors occur. Hence, we denote $\gamma^{k, l} \in[0,1]$, for $k, l \in\{1,2\}$, be the probability that a bi-directional connection between $G_{k}$ and $G_{l}$ be terminated by the peer from the $G_{l}$ side. We use $\gamma^{1,1}$ and $\gamma^{1,2}$ as inputs to derive the possible events that cause the termination of a bi-directional connection in $G_{1}$.

There are three possible processes that can cause a state transition in our Markov chain $\mathcal{M}_{G}$. These processes are: cut process, search process and the match process. Let us describe these processes in detail.

Cut Process: for this process, bi-directional connections are terminated based on $\gamma^{k, l}$. Let us focus on the derivation of $\gamma^{2,1}$ and $\gamma^{2,2}$, with $\gamma^{1,1}$ and $\gamma^{1,2}$ being input values. Define the $\boldsymbol{\delta}^{(2)}=\left\{\delta_{i, j}^{(2)} \mid(i, j) \in S_{G}\right\}$ as the probability vector where $\delta_{i, j}^{(2)}$ is the probability that one of $G_{2}$ peer's bi-directional connection is terminated by a peer in state $(i, j)$, which can be expressed as:

$$
\delta_{i, j}^{(2)}=\sum_{k=u(i, j)}^{N_{1}} \min \left\{\frac{k-u(i, j)}{j}, 1\right\} B\left(N_{1}, \frac{1}{N}, k\right),
$$

where $u(i, j)=K-(i+j)-1$ is the number of idle uploading connections for a peer in state $(i, j)$. Assuming that the number of bi-directional connections are uniformly distributed among peers in the same group, we have,

$$
\gamma^{2,1}=\frac{\sum_{(i, j) \in S_{G}} j \pi_{i, j}^{(1)} \delta_{i, j}^{(2)}}{\sum_{(i, j) \in S_{G}} j \pi_{i, j}^{(1)}} ; \gamma^{2,2}=\frac{\sum_{(i, j) \in S_{G}} j \pi_{i, j}^{(2)} \delta_{i, j}^{(2)}}{\sum_{(i, j) \in S_{G}} j \pi_{i, j}^{(2)}} .
$$

Let $\boldsymbol{Q}_{C}^{(k)}$ be the one-step transition probability matrix of the cut process for a $G_{k}$ peer, where $k \in\{1,2\}$, then the transition probability $\operatorname{Prob}\left\{(i, j) \mid\left(i^{\prime}, j^{\prime}\right)\right\}$ for $\boldsymbol{Q}_{C}^{(k)}$ is:

$$
B\left(i^{\prime}, \gamma^{k, 1}, i^{\prime}-i\right) B\left(j^{\prime}, \gamma^{k, 2}, j^{\prime}-j\right) \mathbf{1}_{\left\{\left(i \leq i^{\prime}\right) \wedge\left(j \leq j^{\prime}\right)\right\}},
$$

with $\left(i^{\prime}, j^{\prime}\right) \in S_{G},(i, j) \in S_{G}$, and $\mathbf{1}_{\{x\}}$ is an indicator function that $\mathbf{1}_{\{x\}}=1$ if condition $x$ is true and 0 otherwise. Search process: for this process, the peer performs optimistic unchoke and randomly selects another peer at the end of each round. We define $\boldsymbol{\alpha}^{(k)}=\left\{\alpha_{i, j}^{(k)} \mid(i, j) \in S_{G}\right\}$ as the probability vector for group $G_{k}, k=\{1,2\}$, where $\alpha_{i, j}^{(k)}$ is the probability that a peer of type $G_{k}$ randomly unchokes and the receiving peer, which is in state $(i, j)$, decides to provide a reciprocative upload. Note that an optimistic unchoke from a peer in group $G_{1}$ have a higher priority than any unchoke operations (both for regular and optimistic) for peers from $G_{2}$. We have:

$$
\alpha_{i, j}^{(1)}=\sum_{k=0}^{N_{1}} \min \left\{\frac{K-i-1}{k+1}, 1\right\} B\left(N_{1}, \frac{1}{N}, k\right) .
$$

On the other hand, optimistic unchokes from a peer in $G_{2}$ are reciprocated only when the peer in $G_{1}$ has an idle upload connection. Therefore, $\alpha_{i, j}^{(2)}$ can be expressed as:

$\alpha_{i, j}^{(2)}=\sum_{k=0}^{u(i, j)} \sum_{l=0}^{N_{2}}\left[\min \left\{\frac{u(i, j)-k}{l+1}, 1\right\} B\left(N_{1}, \frac{1}{N}, k\right) B\left(N_{2}, \frac{1}{N}, l\right)\right]$, with $u(i, j)=K-(i+j)-1$ as mentioned above. Finally, the probability that an optimistic unchoke from a peer in $G_{k}$ receives a reciprocation from a peer in $G_{l}$, which we denote as $\beta^{k, l}$, is:

$$
\beta^{k, l}=\boldsymbol{\pi}^{(k)} \times\left(\boldsymbol{\alpha}^{(l)}\right)^{T} \quad \text { with } k, l=\{1,2\} .
$$

Table I summarizes the one-step transition probability matrix $\boldsymbol{Q}_{S}^{(k)}$ of the search process for peers from group $G_{k}, k=$ $\{1,2\}$.

\begin{tabular}{||c||c||c||}
\hline State & Probability & Condition \\
\hline \hline$(i, j)$ & 1 & $i=K-1, j=0$ \\
\hline$(i, j)$ & $1-\frac{N_{2}}{N} \beta^{k, 2}$ & $i+j=K-1, j>0$ \\
\hline$(i, j)$ & $1-\frac{N_{2}}{N} \beta^{k, 2}-\frac{N_{1}}{N} \beta^{k, 1}$ & $i+j<K-1$ \\
\hline$(i+1, j-1)$ & $\frac{N_{1}}{N} \beta^{k, 1}$ & $i+j=K-1, j>0$ \\
\hline$(i+1, j)$ & $\frac{N_{1}}{N} \beta^{k, 1}$ & $i+j<K-1$ \\
\hline$(i, j+1)$ & $\frac{N_{2}}{N} \beta^{k, 2}$ & $i+j<K-1$ \\
\hline
\end{tabular}

TABLE I

TRANSITION PROBABILITIES OF SEARCH PROCESS WITH INITIAL STATE $(i, j)$

Match process: a peer uses the Greedy Selection algorithm to select contributing peers to unchoke. Let us consider a $G_{1}$ peer, say $v$. Peer $v^{\prime} s$ reciprocation to the optimistic unchoke can establish a bi-directional connection when the peer which initiates the optimistic unchoke is in state $(i, j)$, where $i<$ $K-1$. While a $G_{2}$ peer's reciprocation can be accepted only when the peer which initiates the optimistic unchoke is in state $(i, j)$, where $i+j<K-1$. We define $E_{k, l}$ be the set of candidate peers in group $G_{k}$ which can still establish a bi-directional connection from the point of view of peers in $G_{l}$, then we have:

$$
\begin{aligned}
& E_{k, 1}=\left\{v \mid v \in G_{k}, \text { with state }(i, j), i<K-1\right\}, \\
& E_{k, 2}=\left\{v \mid v \in G_{k}, \text { with state }(i, j), i+j<K-1\right\} .
\end{aligned}
$$

Also, define $\bar{E}_{k, l}=G_{k}-E_{k, l}$ as the complement set of $E_{k, l}$.

Given an initial state, the state transition is determined by the number of received optimistic unchokes from the set $E_{k, 1}, \bar{E}_{k, 1}, E_{k, 2}, \bar{E}_{k, 2}$ respectively. We define $x, y, z, w$, where $x \in\left\{0, . .,\left|E_{k, 1}\right|\right\}, y \in\left\{0, . .\left|\bar{E}_{k, 1}\right|\right\}, z \in\left\{0, . .,\left|E_{k, 2}\right|\right\}$ and $w \in\left\{0, . .,\left|E_{k, 2}\right|\right\}$, as the number of optimistic unchokes from the corresponding sets. Table II illustrates transition probability matrix $\boldsymbol{Q}_{M}^{(k)}$, for $k \in\{1,2\}$. Note that the existing bidirectional connection between $G_{1}$ peers can never be terminated in this process, thus we have the $\operatorname{Prob}\left\{(i, j) \mid\left(i^{\prime}, j^{\prime}\right), i<\right.$ $\left.i^{\prime}\right\}=0$, as shown in the second row of Table II, and the function $\operatorname{Select}(a, b, c, d)$ is:

$$
\operatorname{Select}(a, b, c, d)=\frac{\left(\begin{array}{l}
c \\
a
\end{array}\right)\left(\begin{array}{l}
d \\
b
\end{array}\right)}{\left(\begin{array}{c}
c+d \\
a+b
\end{array}\right)} .
$$

To compute the steady state probability vectors for $\boldsymbol{\pi}^{(1)}$ and $\boldsymbol{\pi}^{(2)}$, we use the following balanced equations:

$\boldsymbol{\pi}^{(k)}=\boldsymbol{\pi}^{(k)} \times \boldsymbol{Q}_{C}^{(k)} \times \boldsymbol{Q}_{S}^{(k)} \times \boldsymbol{Q}_{M}^{(k)} ; \boldsymbol{\pi}^{(k)} \boldsymbol{e}=1$, for $k=\{1,2\}$. 


\begin{tabular}{||c||c||c||}
\hline State & Conditions & Next State \\
\hline \hline$\left(i^{\prime}, j^{\prime}\right)$ & 0 & $(i, j), i<i^{\prime}$ \\
\hline$\left(\begin{array}{c}\left.i^{\prime}, j^{\prime}\right), \\
j^{\prime}>0\end{array}\right.$ & $\left(x=i-i^{\prime}\right) \wedge(y=u(i, j))$ & $(i, j)$, \\
\hline$\left(i^{\prime}, j^{\prime}\right)$, & $\left(x \geq\left(i-i^{\prime}\right)\right) \wedge(y \geq K-i-1) \wedge \operatorname{Select}\left(i-i^{\prime}, K-i-1, x, y\right)$ & $0<j<j^{\prime}$ \\
$j^{\prime}>0$ & & $(i, 0)$, \\
\hline$\left(i^{\prime}, j^{\prime}\right)$ & $\left\{\left(x=i-i^{\prime}\right) \wedge(y \leq u(i, j))\right\} \wedge\left\{\left((w<u(i, j)-y) \wedge\left(z=j-j^{\prime}\right)\right)\right.$ \\
& $\left.\vee\left((w \geq u(i, j)-y) \wedge\left(z \geq j-j^{\prime}\right) \wedge \operatorname{Select}\left(j-j^{\prime}, u(i, j)-y, z, w\right)\right)\right\}$ & $i \geq i^{\prime},\left(j>j^{\prime}\right) \vee\left(j=j^{\prime}>0\right)$ \\
\hline \multirow{2}{*}{$\left(i^{\prime}, 0\right)$} & $\left\{\left(x \geq\left(i-i^{\prime}\right)\right) \wedge(y \geq K-i-1) \wedge \operatorname{Select}\left(i-i^{\prime}, K-i-1, x, y\right)\right\}$ & $(i, 0)$, \\
& $\left\{\left(x=i-i^{\prime}\right) \wedge(y \leq u(i, j))\right\} \wedge\left\{\left((w<u(i, j)-y) \wedge\left(z=j-j^{\prime}\right)\right)\right.$ & $i \geq i^{\prime}$ \\
& $\left.\left.\vee\left((w \geq u(i, j)-y) \wedge\left(z \geq j-j^{\prime}\right) \wedge \operatorname{Select}\left(j-j^{\prime}, u(i, j)-y, z, w\right)\right)\right]\right\}$ & \\
\hline
\end{tabular}

TABLE II

TRANSITION PROBABILITIES FOR A MATCH PROCESS

Note that the above steady state probability vector can be easily found by using standard numerical methods, e.g., power method.

\section{Extending to Multi-Groups}

Extending the above Markov chain to handle a BT-like system with more than two groups of peers can be prohibitive since the state space of the Markov chain will be large. To resolve this problem, we consider the following simplifications. Consider a BT-like system with $n>2$ group of peers, we assume the GS algorithm operates as:

1) For a peer $v$ in group $G_{1}$, besides the bi-directional connections to peers in $G_{1}$, $v$ 's regular unchokes are randomly distribute in $G_{k}$ with $1<k \leq n$, if there are enough receivers in such groups.

2) For a peer $v$ in group $G_{k}$, with $1<k \leq n$, besides $v$ 's bi-directional connections reciprocated to peers in groups $G_{l}$, where $1 \leq l \leq k$, $v$ 's regular unchokes are randomly distribute in $G_{m}$ with $k<m \leq n$ if there are enough receivers in such groups.

With the above assumptions, we construct a discrete time Markov process $\mathcal{M}$ with the state space $S_{R}$ for group $G_{k}$ :

$$
S_{R}=\{i \mid 0 \leq i \leq K-1\}
$$

where $i$ is the number of matched connections, i.e., the bidirectional connections between peers of the same group. We also define the probability vector $\boldsymbol{\pi}^{(k)}=\left\{\pi_{0}^{(k)}, \ldots, \pi_{K-1}^{(k)}\right\}$, where $\pi_{i}^{(k)}$ represents the fraction of $G_{k}$ peers holding $i$ matched connections with $i \in S_{R}$. We can now express the clustering index for peers in $G_{k}$ as:

$$
C_{k}=\frac{1}{K-1} \sum_{l=1}^{K-1} l \pi_{l}^{(k)} .
$$

Consider a $G_{k}$ peer, the optimistic and regular unchokes from a peer in group $G_{l}$, where $1 \leq l<k$, have a higher priority than the unchokes from a $G_{k}$ peer. We define $O_{k}$ $\left(R_{k}\right)$ as the set of such optimistic (regular) unchokes of group $G_{k}$. Obviously, we have $\left|O_{k}\right|=\sum_{l=1}^{k-1} N_{l}$. We can determine the cardinality of $R_{k}$ as:

$\left|R_{k}\right|=\min \left\{N_{k} \sum_{l=1}^{k-1}\left[\frac{N_{l}(K-1)\left(1-C_{l}\right)-\left|R_{l}\right|}{\sum_{m=l}^{n} N_{m}}\right],(K-1) N_{k}\right\}$, where $\left|R_{1}\right|=0$ as group $G_{1}$ has the highest bandwidth. Consider a $G_{k}$ peer, because regular unchokes in $R_{k}$ are uniformly distributed, so each of the connection has the probability of $p=\left|R_{k}\right| /\left(N_{k} \cdot(K-1)\right)$ to respond to regular unchokes from a peer of high index group.

Let us consider group $G_{k}$ for $1 \leq k \leq n$. Similar to the Markov model with two groups, there are three processes that cause the state transition. We describe them as follows.

Cut process: in this process bi-directional connections are terminated. Similar to the derivation of the previous Markov chain, the probability that a a state $i$ peer terminates a bidirection connection of $G_{k}$ is:

$$
\begin{aligned}
\delta_{i}^{(k)=} \sum_{x=0}^{K-i-1} \sum_{y=u(x, i)}^{\left|O_{k}\right|} \min \left\{\frac{y-u(x, i)}{i}, 1\right\} \times \\
B(K-i-1, p, x) B\left(\left|O_{k}\right|, \frac{1}{N}, y\right),
\end{aligned}
$$

where $u(x, i)=K-x-i-1$. Assuming that the bi-directional connections are uniformly distributed among $G_{k}$ peers, we have,

$$
\gamma^{(1)}=\text { input value, } \gamma^{(k)}=\frac{\sum_{i=0}^{K-1} i \pi_{i}^{(k)} \delta_{i}^{(k)}}{\sum_{i=0}^{K-1} i \pi_{i}^{(k)}} \text {, for } k>1 \text {. }
$$

Finally, the transition probability matrix $\boldsymbol{Q}_{C}^{(k)}$ has the following transition probability with $i, i^{\prime} \in S_{R}$

$$
\operatorname{Prob}\left\{i \mid i^{\prime}\right\}=B\left(i^{\prime}, \gamma^{(k)}, i^{\prime}-i\right) 1_{\left\{i \leq i^{\prime}\right\}} \text {. }
$$

Search process: In this process, a peer randomly performs optimistic unchoke to search for available peers within the same group. Let $\beta^{(k)}$, for $1 \leq k \leq n$, be the probability that the optimistic unchoke finds an available peer and gets the reciprocated upload connection. We have:

$$
\beta^{(k)}=\frac{N_{k}}{N} \sum_{i=0}^{K-1} \pi_{i}^{(k)} \alpha_{i}^{(k)}
$$

where,

$$
\begin{aligned}
\alpha_{i}^{(k)}=\sum_{x=0}^{K-i-1} \sum_{y=0}^{u(x, i)} \sum_{z=0}^{N_{k}} \min & \left\{\frac{u(x, i)-y}{z+1}, 1\right\} B(K-i-1, p, x) \\
& \times B\left(\left|O_{k}\right|, \frac{1}{N_{k}}, y\right) B\left(N_{k}, \frac{1}{N}, z\right) .
\end{aligned}
$$


Therefore, the transition probability matrix $\boldsymbol{Q}_{S}^{(k)}$ has the following probability with $i \in S_{R}$ :

$\operatorname{Prob}\{i \mid i\}=1-\beta^{(k)} 1_{\{i \leq K-1\}}, \operatorname{Prob}\{i+1 \mid i\}=\beta^{(k)} 1_{\{i<K-1\}}$.

Match process: in this process, a peer responds to the new optimistic unchokes from peers of the same group. Let us consider a group $G_{k}$ with $1 \leq k \leq n$. Define $(x, y, z)$ for a state $i$ peer, where $x \in\{0, . ., K-i-1\}$ is the number of bi-directional connection between $G_{k}$ and $G_{l}$ with $1 \leq$ $l<k$; while $y \in\left\{0, . .,\left|O_{k}\right|\right\}$ and $z \in\left\{0, . ., N_{k}\right\}$ are the number of optimistic unchokes from $G_{l}(1 \leq l<k)$ and $G_{k}$ respectively. Given the initial state of a peer, the state transition is determined by these three variables. Table III depicts the transition probability matrix $\boldsymbol{Q}_{M}^{(k)}$.

\begin{tabular}{||c||c||}
\hline Transition & Condition \\
\hline \hline$p\{0 \mid 0\}$ & $\{(x \leq K-1) \wedge(y \geq u(0, x))\}$ \\
& $\vee(x+y<K-1) \wedge(z=0)\}$ \\
\hline$p\left\{0 \mid i^{\prime}, i^{\prime}>0\right\}$ & $\left(x \leq u\left(0, i^{\prime}\right) \wedge(y \geq u(0, x))\right.$ \\
\hline$p\left\{i \mid i^{\prime}, i^{\prime}>i>0\right\}$ & $\left(x \leq u\left(0, i^{\prime}\right) \wedge(y=u(x, i))\right.$ \\
\hline$p\left\{i \mid i^{\prime}\right\}$, & $\left\{\left(x+y<u(0, i) \wedge\left(z=i-i^{\prime}\right)\right\}\right.$ \\
$i>i^{\prime} \vee i=i^{\prime}>0$ & $\vee\left\{(x+y=u(0, i)) \wedge\left(z \geq i-i^{\prime}\right)\right\}$ \\
\hline
\end{tabular}

TABLE III

TRANSITION PROBABILITIES OF MATCH PROCESS WITH INITIAL STATE $i^{\prime}$

Similar to the first Markov chain, we obtain the steady state vector $\boldsymbol{\pi}^{(k)}$ using Equation 5.

\section{Performance Evaluation and Validation}

To validate our mathematical models of determing the clustering index of BT-like systems, we develop a discrete event simulator to perform peer selection and the related choking algorithm. Since we are interested in the clustering index of the GS algorithm, we implement this peer selection only and each peers will have some chunks that are of interested by other peers. All peers will arrive to the system at time $t=0$ and they stay in the system forever to exchange chunk.

For our discrete event simulator, we set $N=1000$. The other input parameters to our simulator are:

1) $n$, the number of groups,

2) the number of peers in each group $G_{k}, 1 \leq k \leq n$,

3) $K=R+P, R$ and $P$ is the number of regular and optimistic unchokes for any peer,

4) $\omega$, the number of time slots for each peer to perform the optimistic unchoke operation, where $\omega \in\{1,2, \ldots\}$,

5) $\gamma^{1, k}$ for $1 \leq k \leq n$, the probability of terminating a bi-direction connection of $G_{1}$ by a peer in group $G_{k}$.

In our experiments, we first validate the accuracy of our mathematical models in estimating the clustering index, both for a BT-like system with two groups of peers and for systems with more than two groups of peers. We also carry out experiments to illustrate how one can change the clustering index of a system by varying the controllable system parameters, namely, $R, P$ and $\omega$. This is important since for some applications, e.g., P2P multimedia streaming, we may want to have a lower clustering index so that more resourceful peers may help other less resourceful peers in obtaining a satisfiable viewing service. It is important to point out that we set $R=4, P=1$ in the following experiments, unless we state otherwise.

Experiment 1 (Validating the Two-Groups model): In this experiment, we consider a BT-like system with two different types of peers (or two groups). we set the input parameter $n=2$ and $\omega=1$. Therefore, each peer will perform the optimistic unchoke at that end of every round. In Table IV, we present our mathematical prediction of clustering index, the simulation results of clustering index and the corresponding errors when $\gamma^{1,1}=0.01$. As we can observe, our model is very accurate in predicting the clustering indexes for both peer groups. Also Figure 1 illustrates the clustering index for both groups when we set $\gamma^{1,1}$ is equal to 0.01 or 0.2 . One can conclude that our mathematical model can accurately predict the clustering index of each group in this scenario.

\begin{tabular}{||c||c|c||c|c||c|c||}
\hline \multicolumn{1}{||c||}{ Fraction of } & \multicolumn{2}{c|}{ Model } & \multicolumn{2}{c|}{ Simulation } & \multicolumn{2}{c||}{ Error } \\
\hline$G_{1}$ & $G_{1}$ & $G_{2}$ & $G_{1}$ & $G_{2}$ & $G_{1}$ & $G_{2}$ \\
\hline \hline 0.9 & 0.954 & 0.276 & 0.938 & 0.246 & $1.7 \%$ & $12.1 \%$ \\
\hline 0.7 & 0.949 & 0.516 & 0.935 & 0.490 & $1.5 \%$ & $5.2 \%$ \\
\hline 0.5 & 0.940 & 0.664 & 0.928 & 0.613 & $1.3 \%$ & $8.3 \%$ \\
\hline 0.3 & 0.923 & 0.781 & 0.910 & 0.744 & $1.5 \%$ & $4.9 \%$ \\
\hline 0.1 & 0.856 & 0.896 & 0.853 & 0.850 & $0.3 \%$ & $5.3 \%$ \\
\hline
\end{tabular}

TABLE IV

COMPARING THE NUMERICAL \& SIMULATION RESULTS OF CLUSTERING INDEX, FOR $n=2, \gamma^{1, k}=0.01, k=\{1,2\}$.

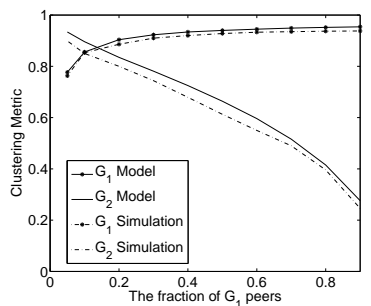

(a) $\gamma^{1,1}=\gamma^{1,2}=0.01$

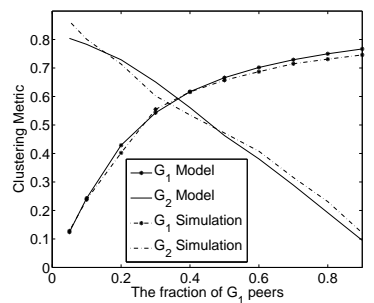

(b) $\gamma^{1,1}=\gamma^{1,2}=0.2$
Fig. 1. Numerical solution vs. simulation result when $n=2$

Experiment 2 (Validating the Multi-Groups model): In this experiment, we verify our mathematical models for multigroups BT-like system. We consider the system has $n=4$ different types of peers (or $G_{1}$ to $G_{4}$ ). Figure 2 illustrates the estimation from our mathematical model and the simulation results by varying the fraction of $G_{4}$ peers in the system. In this experiment, once we set the fraction of $G_{4}$ peers, peers of the other groups are uniformly distributed. In other words, $N_{k}=\frac{N-N_{4}}{3}$ for $k=1,2,3$. We also set $\omega=1$. From Figure 2 , one can observe that the estimation of $G_{1}$ is accurate. While there is a small difference between the mathematical prediction of $G_{k}$, for $k=2,3,4$, with that of the simulation result. This is due to our simplification assumption that the bi-directional connections from $G_{k}$ to $G_{l}$ are uniformly distributed for 
$k<l$. Nevertheless, our mathematical is still quite accurate in estimating the clustering index of other groups.

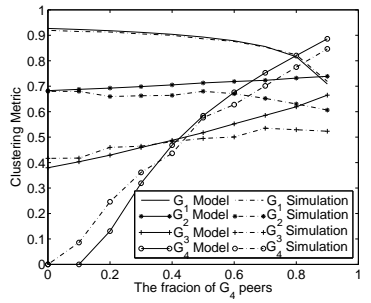

(a) $\gamma^{1, k}=0.01, k=\{1, . ., 4\}$

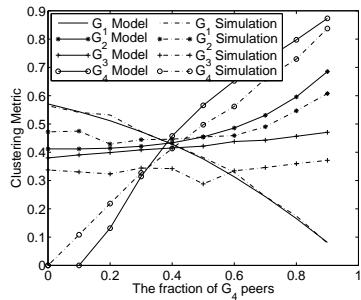

(b) $\gamma^{1, k}=0.2, k=\{1, . ., 4\}$
Fig. 2. Numerical solutions vs. simulation results when $n=4$

Experiment 3 (Varying the clustering index via $\omega$ ): In this experiment, we consider how one can varying the clustering index by changing the value of $\omega$. In Fig. 3, we vary the parameter $\omega$ to $1,2,4,8$. One can observe that the clustering index of $G_{1}$ decrease when the $\omega$ is larger. Since $\omega$ is increased, it will take $G_{1}$ peers longer time to find a more resourceful peer via optimistic unchoke. On the other hand, the clustering index of $G_{2}$ persists as the average number of optimistic unchoke from $G_{1}$ remains relatively unchanged, independent on the values of $\omega$.

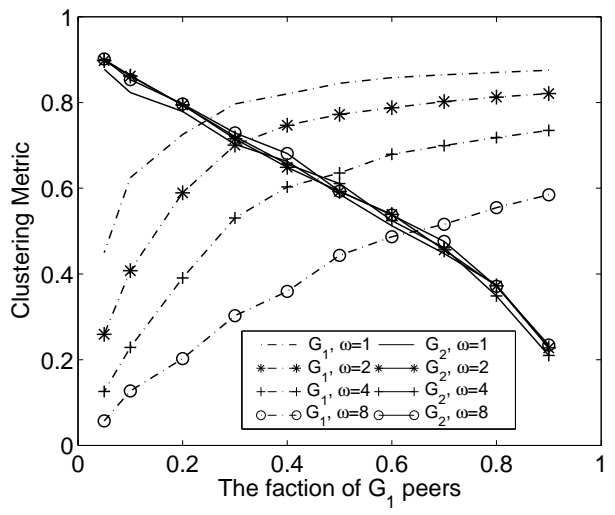

Fig. 3. varying the $\omega, n=2, \gamma^{1,1}=\gamma^{1,2}=0.05$.

Experiment 4 (Varying the clustering index via $R$ and $P$ ): In this experiment, we consider the effect of parameter $R$ and $P$, i.e. the number of regular and optimistic unchokes. We set $\omega=1$. In Fig. 4, we fix $P=1$ and vary the number of regular unchokes $R$. We show that the clustering index of $G_{2}$ increases when $R$ becomes larger. We know that a high clustering index of $G_{1}$ only gives optimistic unchokes to $G_{2}$ which is regardless of reciprocation. So the fraction of optimistic unchokes from $G_{1}$, which have a higher priority, becomes smaller compares to the regular unchokes when $R$ is larger. Therefore, more $G_{2}$ regular unchokes are responding to their compeers, instead of responding to the optimistic unchoke from $G_{1}$. In Fig. 5, we fix $R=4$ and vary the number of optimistic unchokes $P$ to $1,2,4$. The clustering index of $G_{2}$ decrease as more optimistic unchokes from the peers in $G_{1}$ will select the peers in $G_{2}$.

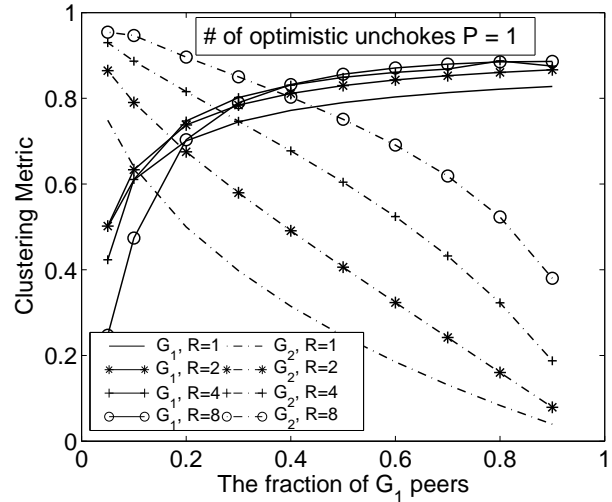

Fig. 4. Varying the $R, n=2, \omega=1, \gamma^{1,1}=\gamma^{1,2}=0.05$.

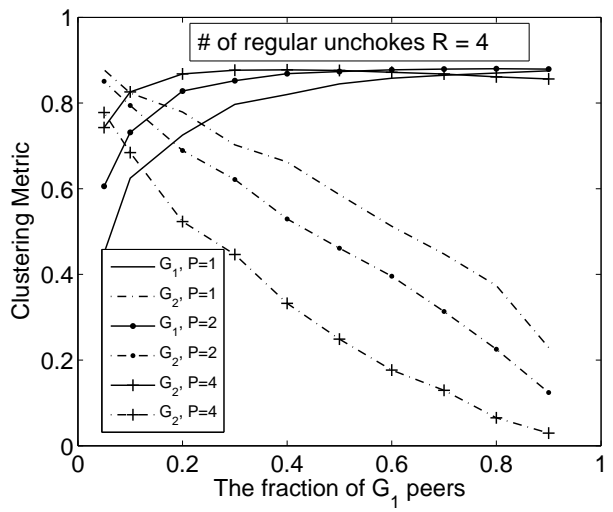

Fig. 5. varying the $P, n=2, \omega=1, \gamma^{1,1}=\gamma^{1,2}=0.05$.

\section{Conclusion}

In this paper, we illustrate that BT protocol will generate a high clustering index system, i.e., peers will connect with other peers which are of similar bandwidth type. We provide two analytical models to accurate evaluate the clustering index of a BT-like system. The models are validated by extensive simulation. We also introduce design knobs to control clustering index so that BT-like streaming systems will have much better performance.

\section{REFERENCES}

[1] B. Cohen, Incentives build robustness in bittorrent, P2P Economics Workshop, Berkeley, CA, 2003.

[2] D. Qiu and R. Srikant, Modeling and performance analysis of BitTorrentlike peer-to-peer networks, ACM SIGCOMM, Portland, Oregon, USA, August 2004.

[3] A. Legout, N. Liogkas, E. Kohler and L. Zhang, Clustering and shar ing incentives in BitTorrent systems, ACM SIGMETRICS, San Diego, California, USA, June, 2007

[4] F. Clvenot-Perronnin, P. Nain and K. W. Ross, Multiclass P2P networks: Static resource allocation for service differentiation and bandwidth diversity, IFIP WG7.3 Performance, Juan-les-Pins, France, 2005.

[5] M.H. Lin, B. Fan, J.C.S. Lui and D.M. Chiu, Stochastic analysis of fileswarming systems, IFIP WG7.3 Performance, German, 2007.

[6] B. Fan, D.M. Chiu and J.C.S. Lui, The Delicate Tradeoffs in BitTorrentlike File Sharing Protocol Design, ICNP, 2006. 